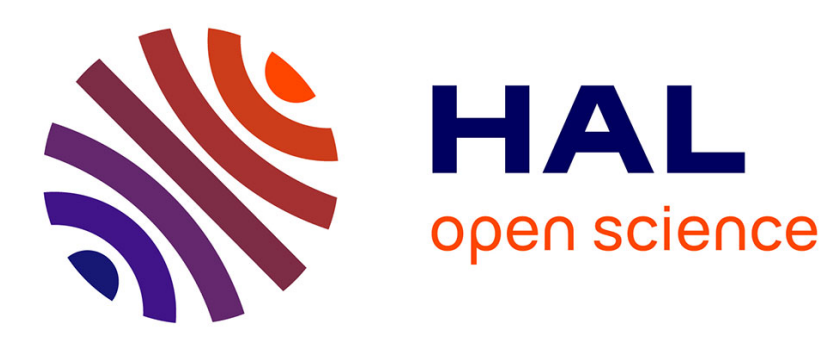

\title{
Modélisation simplifiée 3D du comportement dynamique de structures en béton armé
}

X. H. Nguyen, J. Mazars, Panagiotis Kotronis

\section{To cite this version:}

X. H. Nguyen, J. Mazars, Panagiotis Kotronis. Modélisation simplifiée 3D du comportement dynamique de structures en béton armé. Revue Européenne de Génie Civil, 2006, 10 (3), pp.361-374. 10.1080/17747120.2006.9692833 . hal-01007076

\section{HAL Id: hal-01007076 https://hal.science/hal-01007076}

Submitted on 5 Nov 2019

HAL is a multi-disciplinary open access archive for the deposit and dissemination of scientific research documents, whether they are published or not. The documents may come from teaching and research institutions in France or abroad, or from public or private research centers.
L'archive ouverte pluridisciplinaire HAL, est destinée au dépôt et à la diffusion de documents scientifiques de niveau recherche, publiés ou non, émanant des établissements d'enseignement et de recherche français ou étrangers, des laboratoires publics ou privés. 


\section{Modélisation simplifiée 3D du comportement dynamique de structures en béton armé}

\section{Xuân-Huy Nguyen — Jacky Mazars — Panagiotis Kotronis}

Laboratoire Sols, Solides, Structures

INP Grenoble, Université Joseph Fourrier, CNRS/ RNVO

Domaine Université, BP 53

F-38041 Grenoble cedex 9

Nguyen@geo.hmg.inpg.fr

\{Jacky.Mazars, Panagiotis.Kotronis\}@.inpg.fr

RÉSUMÉ. Dans le cadre du programme européen ECOLEADER, des essais ont été réalisés sur la table sismique du laboratoire LNEC à Lisbonne. La structure testée est représentative d'un bâtiment à 5 niveaux à murs banchés porteurs comportant un mur à file d'ouvertures. Le programme expérimental a comme objectif l'évaluation du comportement de ce type de structure soumis à des chargements bidirectionnels. Des éléments poutres multifibres sont utilisés pour la modélisation de la structure. Les modèles de comportement utilisés associent la plasticité pour les aciers et l'endommagement pour le béton. La comparaison des résultats numériques avec l'expérience montre la pertinence de l'approche.

ABSTRACT. In the framework of the ECOLEADER program dynamic tests have been performed on the seismic table at the laboratory LNEC in Lisbon. The structure tested is a five floor building composed of two parallel walls linked with a perpendicular one that has openings. The scope of the experimental program is to evaluate the behaviour of this structure under bi-directional loading. Multifiber beams are used for the numerical model of the structure. Constitutive laws are based on plasticity for steel and damage mechanics for concrete. Comparison of the numerical and the experimental results shows the performance of the approach.

MOTS-CLÉS : séismes, poutre multifibre, endommagement, murs en béton armé.

KEYWORDS: earthquakes, multifiber beam, damage mechanics, RC structure wall. 


\section{Introduction}

Dans le but d'étudier et de valider le comportement de murs en béton armé sous sollicitation sismique, plusieurs programmes de recherche ont été réalisés ces dernières années. Le projet CASSBA (Conception et Analyse Sismique des Structures en Béton Armé, Brachet et al., 1994) a démontré le bon fonctionnement de murs faiblement armés, simplement posés sur une table vibrante. Le programme CAMUS (Conception et Analyse des MUrs sous séisme, Coin, 2000 ; CAFEELECOEST/ICONS, 2001) s'inscrivait dans le cadre de la maîtrise du risque sismique pour des murs en béton armé dimensionnés selon l'Eurocode 8 ou les règles PS92, simplement posés sur une couche de sable ou encastrés sur la table sismique et sollicités dans leur plan. Le programme suivant, CAMUS 2000 (Bisch et al., 2000), voulait démontrer la légitimité de la conception «mur faiblement armé » pour des réponses 3D. Des résultats en bidirectionnel ont pu être obtenus sur l'essai CAMUS 2000-1 mais le contreventement métallique s'est avéré ne pas être représentatif d'un contreventement classique béton. La structure la plus représentative d'une cellule contreventée en béton est celle utilisée dans le nouveau programme ECOLEADER (Bisch et al., 2005 ; Kotronis et al., 2005b ; Mazars et al., 2005) à Lisbonne. C'est donc sur les résultats de cet essai que nous avons réalisé les présentes études.

Le programme ECOLEADER concerne des essais de murs faiblement armés testés sur la table sismique du LNEC (National Laboratory for Civil Engineering) à Lisbonne. Il s'agit d'une étude comparée du comportement de deux maquettes à murs banchés porteurs et à coffrage identique et comportant un mur à file d'ouvertures. La première maquette a été conçue par l'Université de Ljubljana en Slovénie, selon les usages en Europe centrale. Le dimensionnement de la deuxième maquette a été faite par des experts du génie parasismique suivant le concept français de murs structuraux faiblement armés. Les résultats que nous présentons dans cet article concernent la deuxième maquette.

\section{Elément poutre multifibre}

L'analyse de structures soumises à des chargements dynamiques nécessite des stratégies de modélisation capables de représenter le comportement non linéaire jusqu'à la ruine. Ces dernières peuvent être classées selon deux groupes :

- des stratégies détaillées, fondées sur la mécanique du solide et une description du comportement des matériaux au niveau local,

- des stratégies fondées sur une discrétisation globale.

Dans la première catégorie - qui nous intéresse plus particulièrement dans ce travail - nous trouvons des modèles «classiques » aux éléments finis où la structure est modélisée par des éléments de milieu continu 2D ou 3D et des modèles de type « fibre» ayant un élément de type poutre comme support. Ce type de description possède les avantages d'hypothèses simplificatrices associées à une cinématique de 
type poutre Euler-Bernoulli (Owen et al., 1980 ; La Borderie, 1991 ; Ghavamian et al., 1998 ; Ghavamian et al., 2002) ou Timoschenko (Guedes et al., 1994 ; Dubé, 1997, Petrangeli et al., 1999 ; Kotronis et al., 2004, 2005a ; Mazars et al., 2006) tout en offrant une solution pratique et efficace pour une analyse non linéaire complexe de structures composites (par exemple en béton armé).

\section{Description de la maquette ECOLEADER}

La maquette testée est à l'échelle $1 / 3$ et possède une masse de 31,6 tonnes. Elle est composée de deux murs porteurs liés par six planchers et une longrine inférieure ancrée sur la table sismique afin de reproduire une liaison de type encastrement. De plus, elle est contreventée dans le sens perpendiculaire aux murs par un mur intérieur avec ouvertures. Les murs ont chacun une hauteur de 4,5 m (plus 0,6 m la longrine), une longueur de $1,60 \mathrm{~m}$ et une épaisseur de $6 \mathrm{~cm}$. La longrine inférieure a une longueur de $2 \mathrm{~m}$, une hauteur de $0,6 \mathrm{~m}$ et une épaisseur de $20 \mathrm{~cm}$. Les principales caractéristiques de la maquette sont données à la figure 1 .
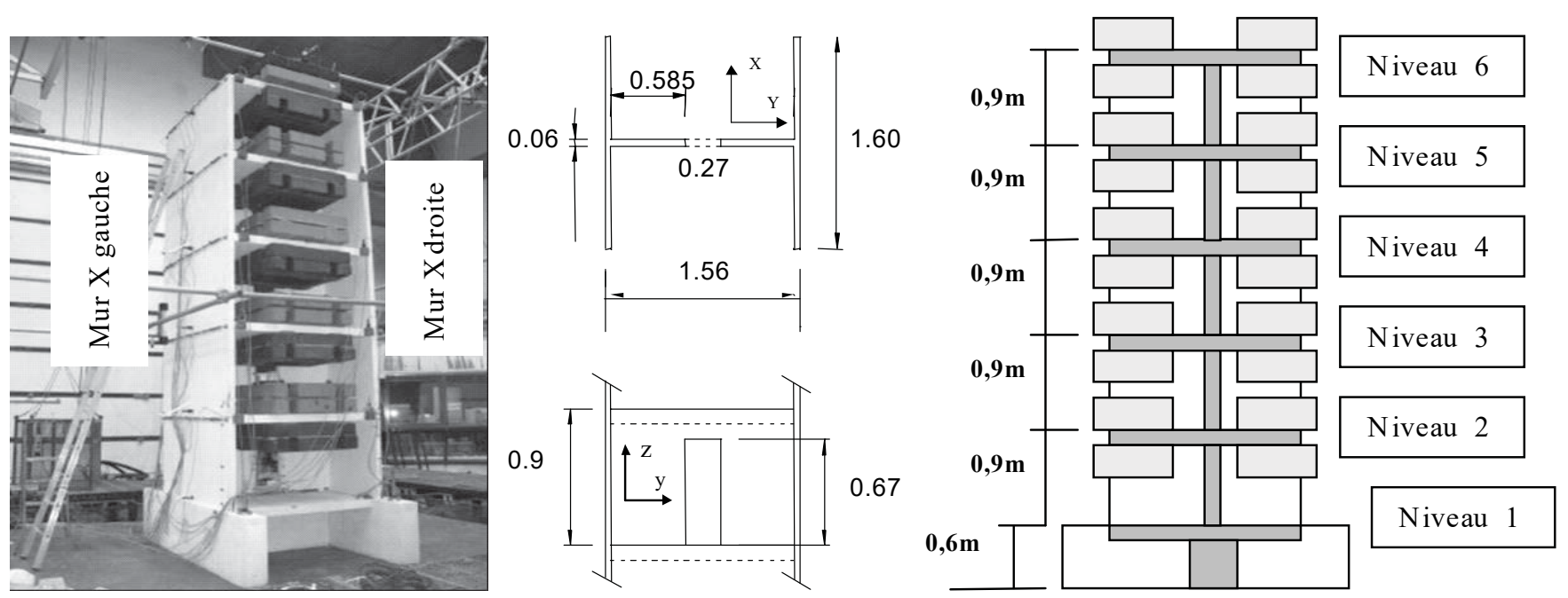

Figure 1. Maquette ECOLEADER. Description de la structure (dimensions en m)

Des masses additionnelles ont été ajoutées à la partie supérieure et inférieure de chaque plancher, sauf celui du niveau 1.

\begin{tabular}{|c|c|c|}
\hline Niveau & Masses additionnelles & Hauteur \\
\hline 6 & $4800 \mathrm{~kg}$ & $5,05 \mathrm{~m}$ \\
\hline 5 & $4800 \mathrm{~kg}$ & $4,16 \mathrm{~m}$ \\
\hline 4 & $4800 \mathrm{~kg}$ & $3,26 \mathrm{~m}$ \\
\hline 3 & $4800 \mathrm{~kg}$ & $2,36 \mathrm{~m}$ \\
\hline 2 & $4800 \mathrm{~kg}$ & $1,46 \mathrm{~m}$ \\
\hline
\end{tabular}

Tableau 1. Maquette ECOLEADER. Positions et valeurs des masses additionnelles 
Les armatures de la maquette sont présentées dans le tableau 2. Les niveaux 1 et 2 sont davantage armés que les niveaux supérieurs.

\begin{tabular}{|c|c|c|}
\hline Niveau & $\begin{array}{c}\text { Armatures verticales du mur } \\
\text { selon Y }\end{array}$ & $\begin{array}{c}\text { Armatures verticales des murs } \\
\text { selon X }\end{array}$ \\
\hline $5-6$ & $2 \Phi 6=0,57 \mathrm{~cm}^{2}$ & $1 \Phi 4,5=0,159 \mathrm{~cm}^{2}$ \\
\hline $4-5$ & $2 \Phi 4,5+2 \Phi 6=0,88 \mathrm{~cm}^{2}$ & $1 \Phi 4,5=0,159 \mathrm{~cm}^{2}$ \\
\hline $3-4$ & $3 \Phi 4,5+2 \Phi 6=1,04 \mathrm{~cm}^{2}$ & $2 \Phi 4,5=0,318 \mathrm{~cm}^{2}$ \\
\hline $2-3$ & $4 \Phi 4,5+2 \Phi 6=1,20 \mathrm{~cm}^{2}$ & $2 \Phi 5+1 \Phi 4,5=0,561 \mathrm{~cm}^{2}$ \\
\hline $1-2$ & $4 \Phi 4,5+2 \Phi 6=1,20 \mathrm{~cm}^{2}$ & $2 \Phi 5+3 \Phi 4,5=0,869 \mathrm{~cm}^{2}$ \\
\hline
\end{tabular}

Tableau 2. Maquette ECOLEADER. Ferraillage

\section{Présentation du modèle numérique}

\subsection{Discrétisation}

Des éléments poutres de cinématique Euler-Bernoulli sont utilisés pour la discrétisation spatiale de la structure. La maquette est considérée comme un portique multi-étage dont des poutres horizontales (section en T) représentent les planchers et des poutres verticales (également en T) les murs.
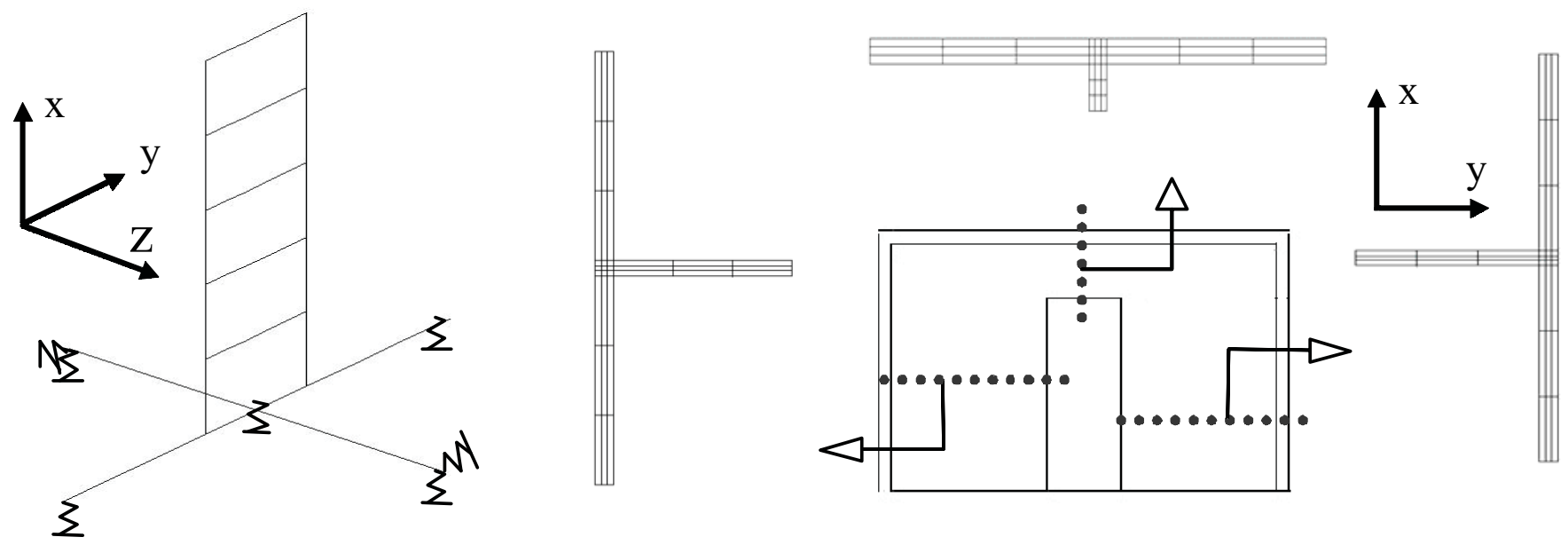

Figure 2. Maquette ECOLEADER. Discrétisation spatiale

Au total le modèle possède 80 éléments poutres avec 2 points de Gauss par élément. Chaque section comporte 36 fibres (figure 2). Les masses additionnelles sont distribuées au niveau de chaque plancher.

Pour reproduire la flexibilité de la table sismique, sept ressorts linéaires (cinq ressorts verticaux et deux horizontaux) sont utilisés (Nguyen et al., 2005 ; Mazars et al., 2005). Les valeurs de ces ressorts ont été calibrées afin de reproduire les 
fréquences et modes propres de la structure (voir section 6.1). La table elle-même est modélisée par des poutres horizontales (infiniment raides dans leurs plans).

\subsection{Lois de comportement des matériaux}

\subsubsection{Béton}

Le modèle «béton » est basé sur la mécanique de l'endommagement (Mazars, 1986 ; La Borderie, 1991). Il est adapté à la description du comportement généré par la création de micro fissures (diminution de la raideur) et du fonctionnement lié à leur re-fermeture au cours des cycles. Les principales caractéristiques du modèle sont :

- utilisation de deux variables d'endommagement (l'une en traction et l'autre en compression),

- prise en compte des déformations permanentes liées à l'endommagement,

- gestion de l'ouverture et de re-fermeture des fissures en introduisant une restauration progressive de la raideur.

Les propriétés du béton utilisées pour les calculs sont: le module d'Young $\mathrm{E}=25000 \mathrm{MPa}$, résistance en compression $\mathrm{fc}=41,7 \mathrm{MPa}$ et résistance en traction $\mathrm{ft}=3,5 \mathrm{MPa}$.

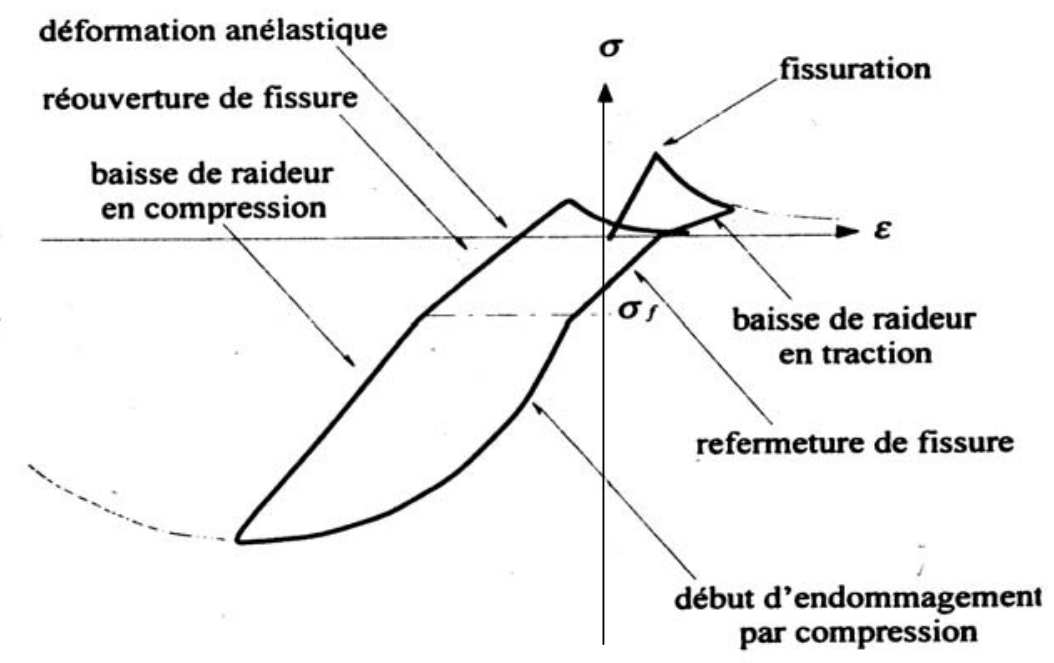

Figure 3. Réponse du modèle utilisé pour le béton (sollicitation de tractioncompression uniaxiale)

\subsubsection{Acier}

Les armatures sont représentées par un modèle élasto-plastique avec écrouissage cinématique linéaire. Les propriétés des aciers utilisées pour les calculs sont: 
module de Young E = $197000 \mathrm{MPa}$, limite d'élasticité $660 \mathrm{MPa}$, la pente de la partie plastique $30000 \mathrm{MPa}$.

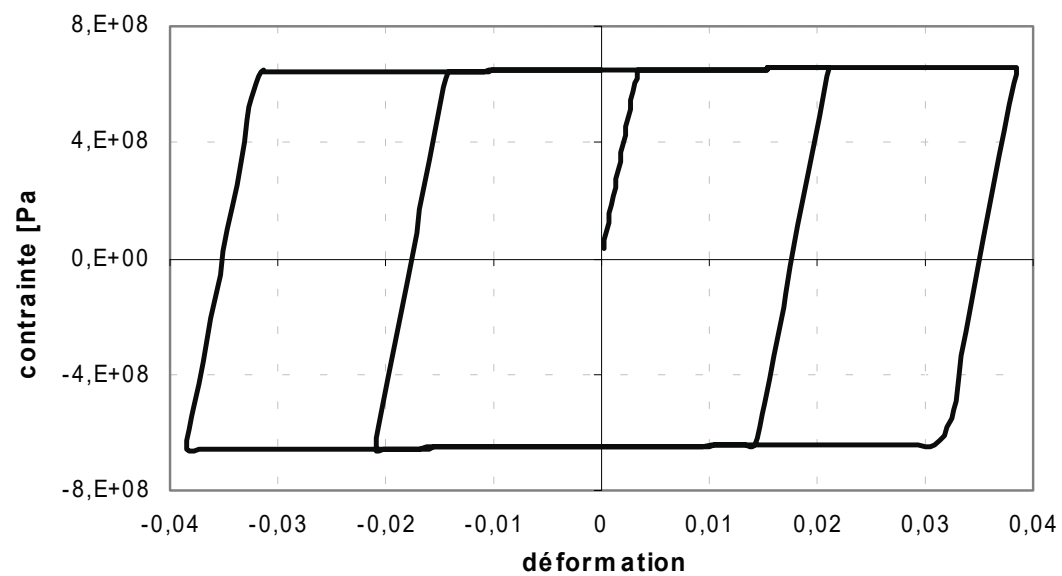

Figure 4. Réponse cyclique du modèle utilisé pour l'acier

\section{Chargement sismique}

Une série d'accélérogrammes (T0 à T6) a été appliquée à la maquette suivant la direction $\mathrm{X}$ et la direction $\mathrm{Y}$. Des signaux naturels de type séisme proche (séisme Tolmezzo-Italie) ont été utilisés. L'histoire des chargements appliqués est donnée cidessous :

\begin{tabular}{|c|c|c|}
\hline Tests & Direction X & Direction $\mathrm{Y}$ \\
\hline $\mathrm{T} 0$ & $0,3 \mathrm{~g}$ & 0 \\
\hline $\mathrm{T} 1$ & 0 & $0,14 \mathrm{~g}$ \\
\hline $\mathrm{T} 2$ & $0,24 \mathrm{~g}$ & $0,13 \mathrm{~g}$ \\
\hline $\mathrm{T} 3$ & $0,45 \mathrm{~g}$ & $0,27 \mathrm{~g}$ \\
\hline $\mathrm{T} 4$ & $0,55 \mathrm{~g}$ & $0,3 \mathrm{~g}$ \\
\hline $\mathrm{T} 5$ & $0,74 \mathrm{~g}$ & $0,36 \mathrm{~g}$ \\
\hline $\mathrm{T} 6$ & $0,85 \mathrm{~g}$ & $0,5 \mathrm{~g}$ \\
\hline
\end{tabular}

Tableau 3. Maquette ECOLEADER. Programme de chargement

\section{Comparaison modèle/expérience}

\subsection{Analyse modale}

Une analyse modale est d'abord effectuée afin d'accéder aux modes propres de la structure. Le tableau 4 présente les valeurs des fréquences et les principaux modes propres de la structure comparés aux valeurs expérimentales. 
Nous pouvons constater une bonne reproduction de la deuxième fréquence propre tandis qu'aucune comparaison ne peut être faite pour la première et la troisième (la valeur expérimentale de la première fréquence n'était pas fiable et la fréquence de torsion n'a pas été mesurée).

\begin{tabular}{|c|c|c|c|}
\hline \multicolumn{1}{|c|}{ Modèle } & Mode 1 (direction $\mathrm{X})$ & Mode 2 (direction $\mathrm{Y})$ \\
\hline $\begin{array}{l}\text { Modèle } \\
\text { simplifié }\end{array}$ \\
\hline
\end{tabular}

Tableau 4. Maquette ECOLEADER. Fréquences et modes propres de la structure

\subsection{Réponse dynamique}

Pour les calculs présentés ci-dessous un amortissement Rayleigh de $1 \%$ sur les deux premières modes est utilisé. Le confinement n'est pas pris en compte et la liaison acier-béton est considérée parfaite. Les figures 5 à 8 montrent les résultats numériques et expérimentaux des déplacements relatifs en tête de la maquette dans les deux directions horizontales (direction $\mathrm{X}$ et $\mathrm{Y}$ ) pour le test T3 (correspondant au niveau de dimensionnement) et le test T6 (le dernier test effectué). Le modèle numérique prévoit que des aciers se rompent à la fin du niveau T3. Pour les niveaux de chargement supérieurs ces aciers ne jouent donc aucun rôle.

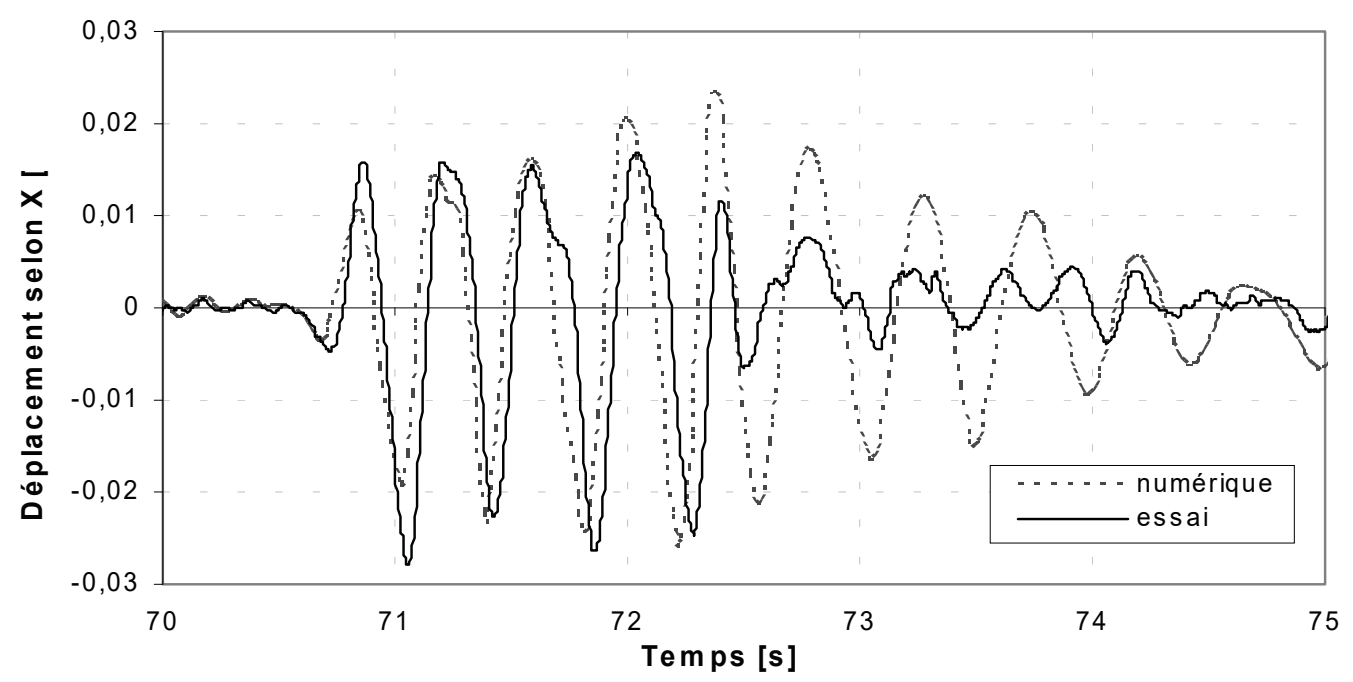

Figure 5. Maquette ECOLEADER. Mur X gauche - Déplacement relatif en tête selon $X$ (test T3) 


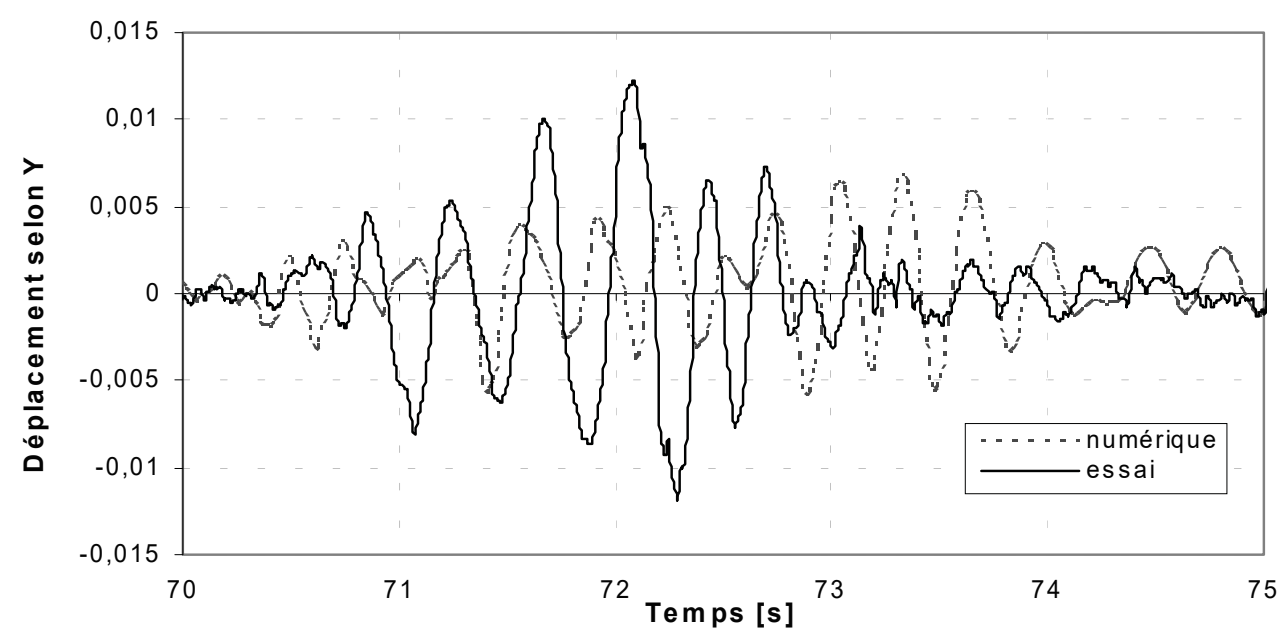

Figure 6. Maquette ECOLEADER. Mur X gauche - Déplacement relatif en tête selon $Y$ (test T3)

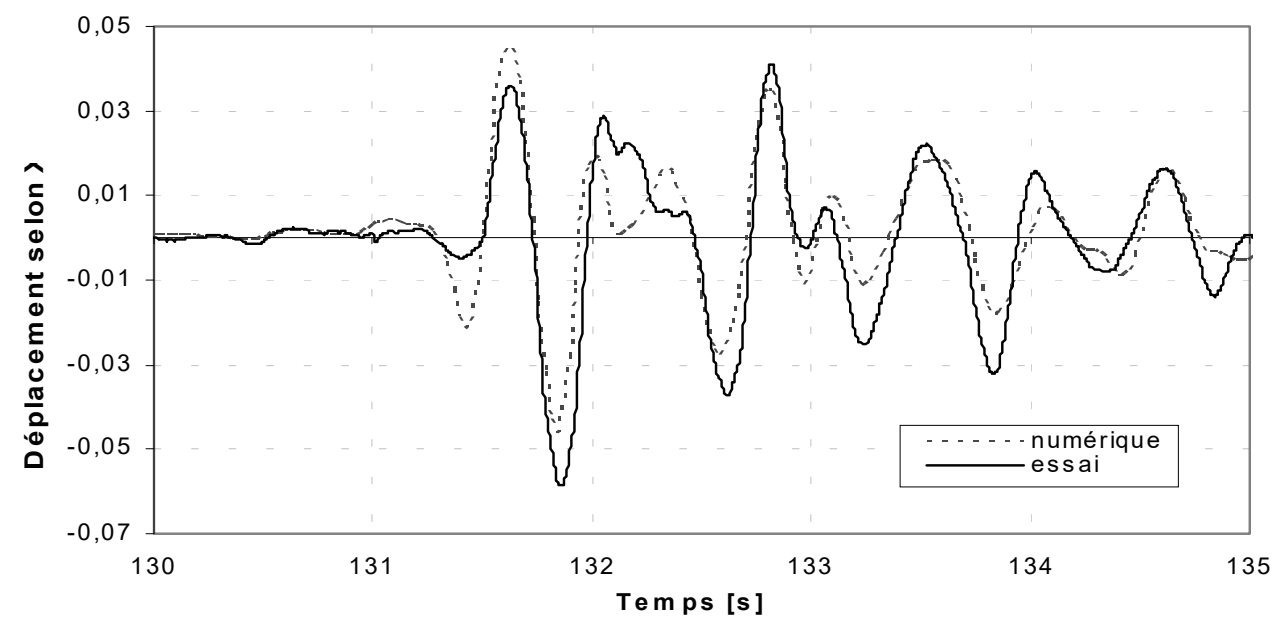

Figure 7. Maquette ECOLEADER. Mur X gauche - Déplacement relatif en tête selon $X$ (test T6)

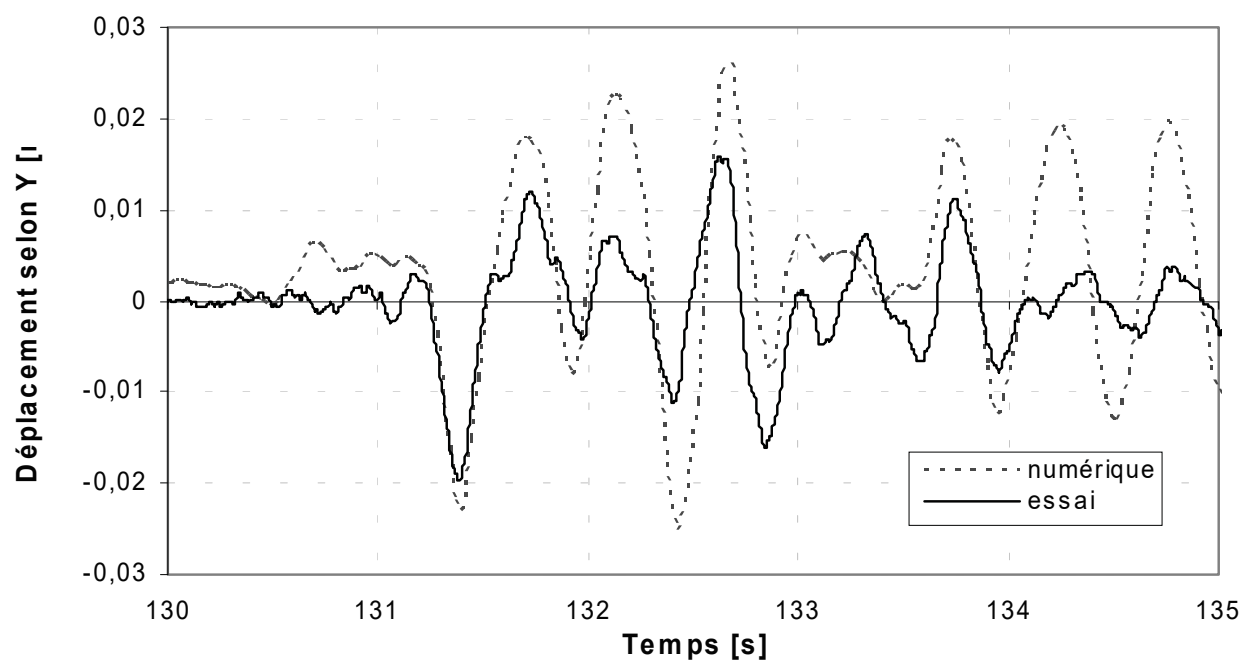

Figure 8. Maquette ECOLEADER. Mur X gauche - Déplacement relatif en tête selon Y (test T6) 
De ces comparaisons nous pouvons noter une bonne prédiction du comportement dans la direction $\mathrm{X}$. Les valeurs maximales et les cycles de réponses calculés sont correctement reproduits. Néanmoins et ceci pour l'ensemble des niveaux les résultats en $\mathrm{Y}$ ne sont pas si satisfaisants. Cette différence de performance dans les deux directions (que d'ailleurs apparaît aussi en utilisant des modèles éléments finis complets en 3D, voir Mazars et al., 2005) est certainement due à un comportement plus complexe dans la direction Y (cisaillement dans les linteaux et les trumeaux qui n'est pas pris en compte par le modèle, influence de la torsion des planchers...)

L'état de dégradation de la structure à la fin des essais est représenté par la carte d'endommagement de traction pour le béton. L'indicateur d'endommagement varie normalement entre 0 et 1 . En filtrant ces valeurs entre 0,95 et 1 , nous faisons apparaître les zones concernées par la macro-fissuration : les deux premiers niveaux ce qui fut constaté durant l'expérimentation. De plus, les murs en $\mathrm{X}$ sont plus endommagés que le mur en Y, ce qui s'est aussi constaté pendant les essais.
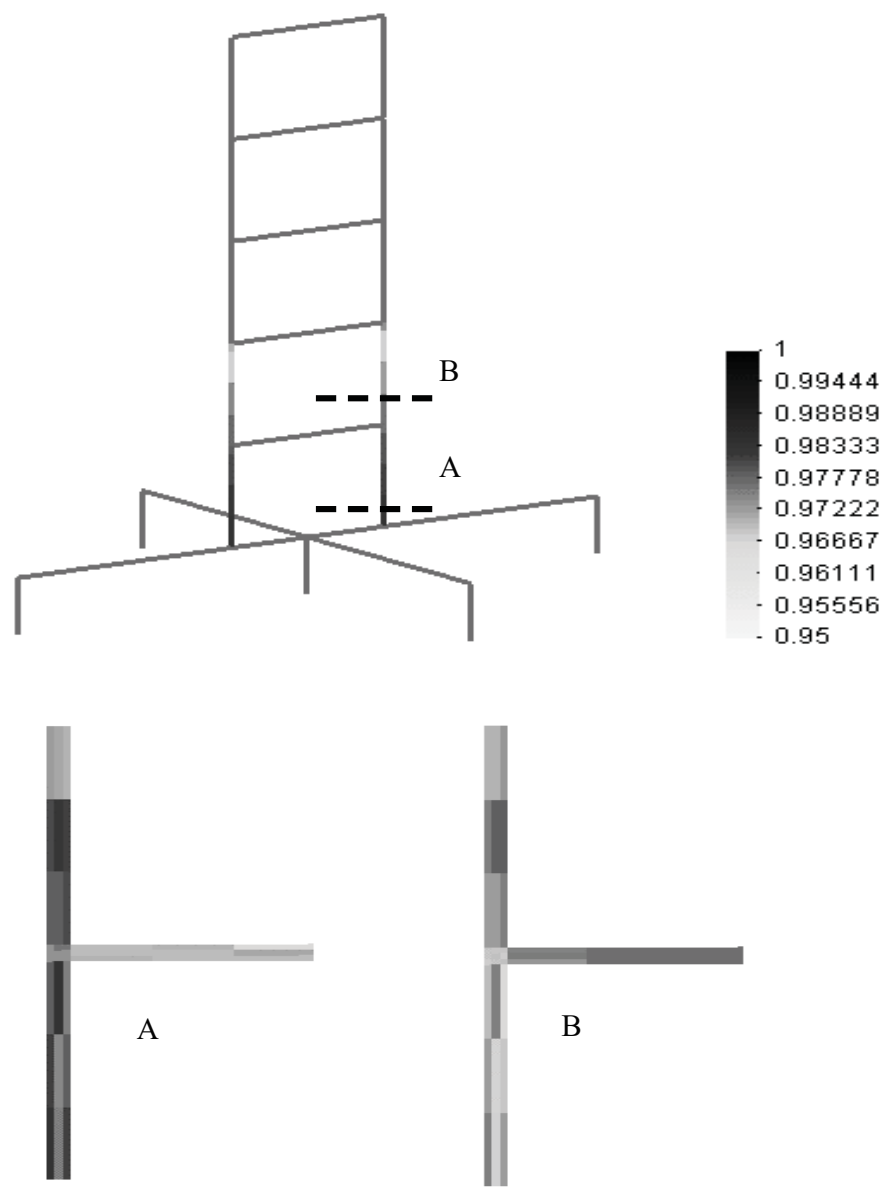

Figure 9. Maquette ECOLEADER. Carte d'endommagement en traction (test T6)

La figure 10 illustre l'état de dégradation à la fin de T6. La structure est fortement endommagée et plusieurs fissures sont concentrées à sa base (niveau 1). Le béton est écaillé dans les extrémités des murs et quelques aciers sont cassés. La comparaison avec la position des fissures prédite par le modèle montre l'efficacité de la stratégie de simulation choisie à prédire les zones critiques. 

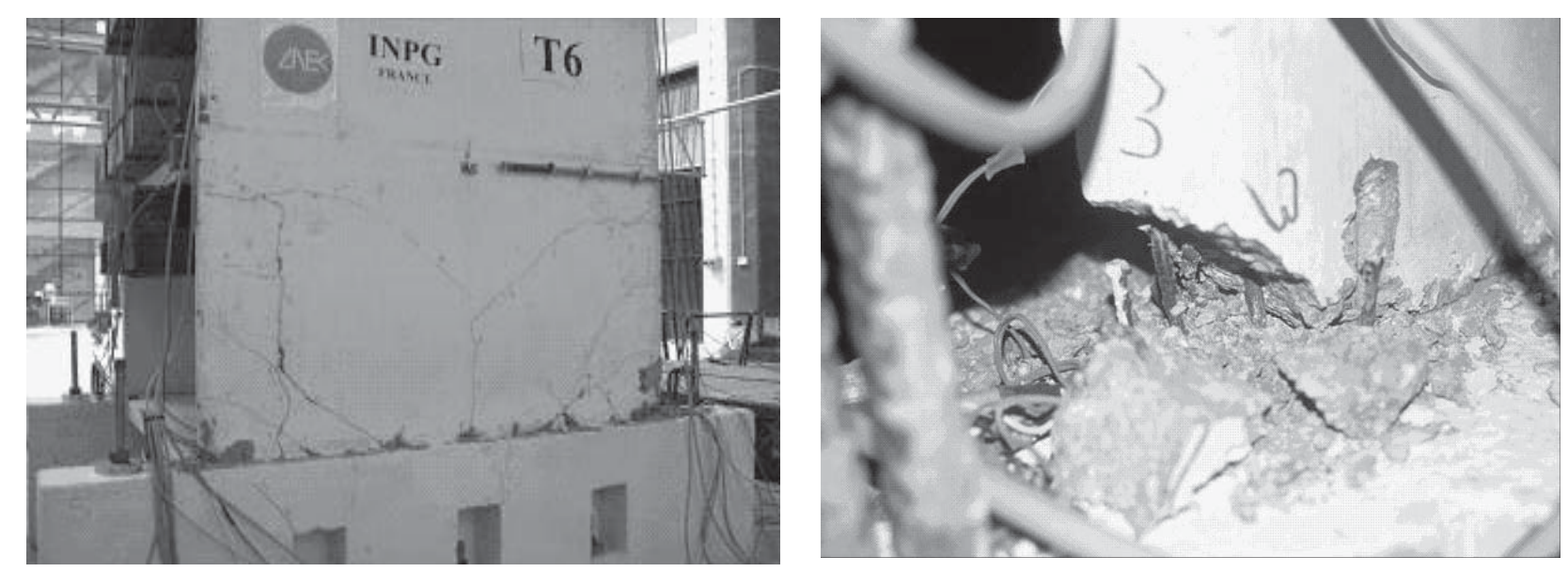

Figure 10. Maquette ECOLEADER. L'état de dégradation (test T6)

Une autre façon de voir la dégradation de la structure est de présenter les déformations des aciers prédites par le modèle. Dans la figure 11, la déformation dans un acier situé sur la fibre extrême est tracée en fonction de la hauteur de la maquette.
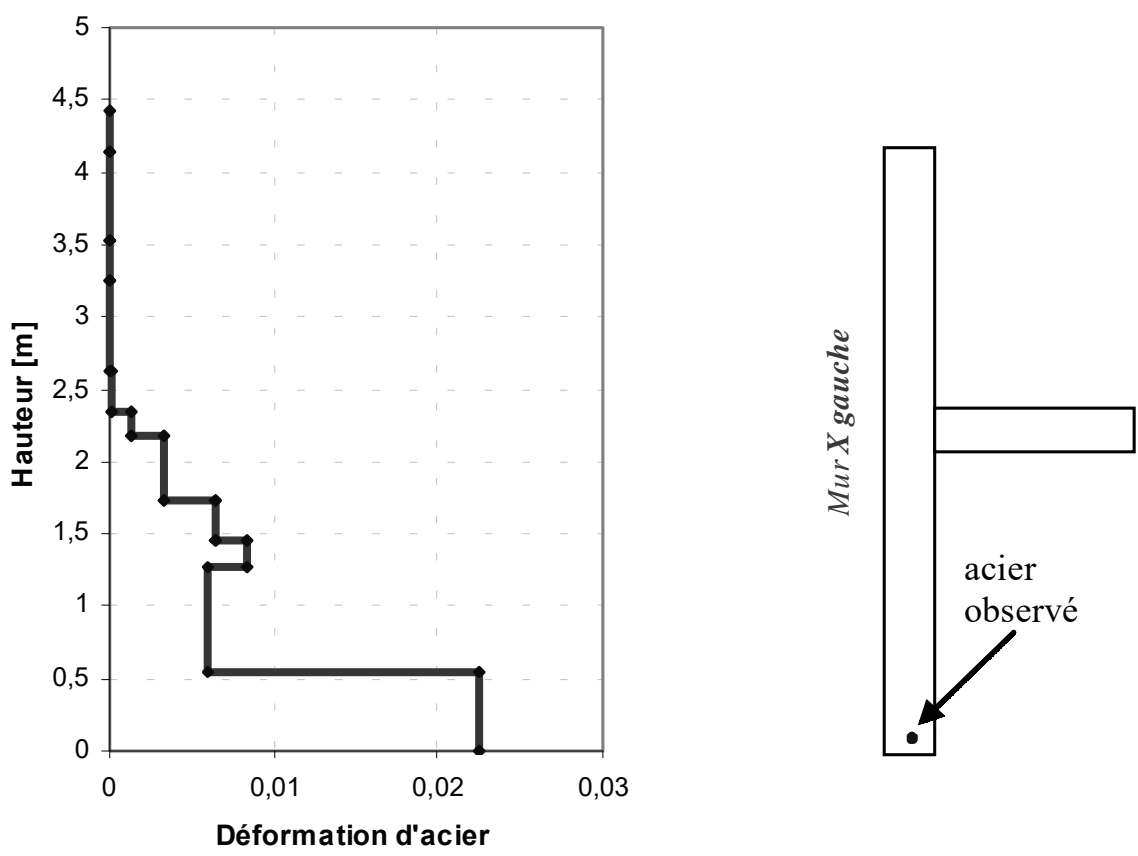

Figure 11. Maquette ECOLEADER. Déformation de l'acier (test T6)

La déformation maximale positive atteint une valeur importante $(>2 \%)$ qui dépasse largement la valeur expérimentale correspondant à la ruine des aciers (environ $1.6 \%$ ) mesurée lors d'essais préliminaires. Un acier cassé par traction à la base de la maquette à la fin de test T6 est visible dans la figure 10 .

Dans les figures 12 et 13 l'ensemble des valeurs (M, N) numériques est présenté en comparaison avec les courbes d'interaction ELU. Nous constatons que les couples $(\mathrm{M}, \mathrm{N})$ correspondants aux murs $\mathrm{X}$ gauche sont positionnés entre les courbes 
ELU et cela de façon assez serrée pour le niveau T3. Pour le dernier niveau de chargement (T6), le modèle numérique prévoit la rupture de la section due aux importantes déformations de compression qui conduisent à l'écrasement du béton et au flambement des aciers. Ce comportement fut aussi constaté lors des essais : Pendant le test T3 un acier s'est rompu à cause de flambement et à la fin de T6 le béton aux extrémités des murs en X s'est éclaté.

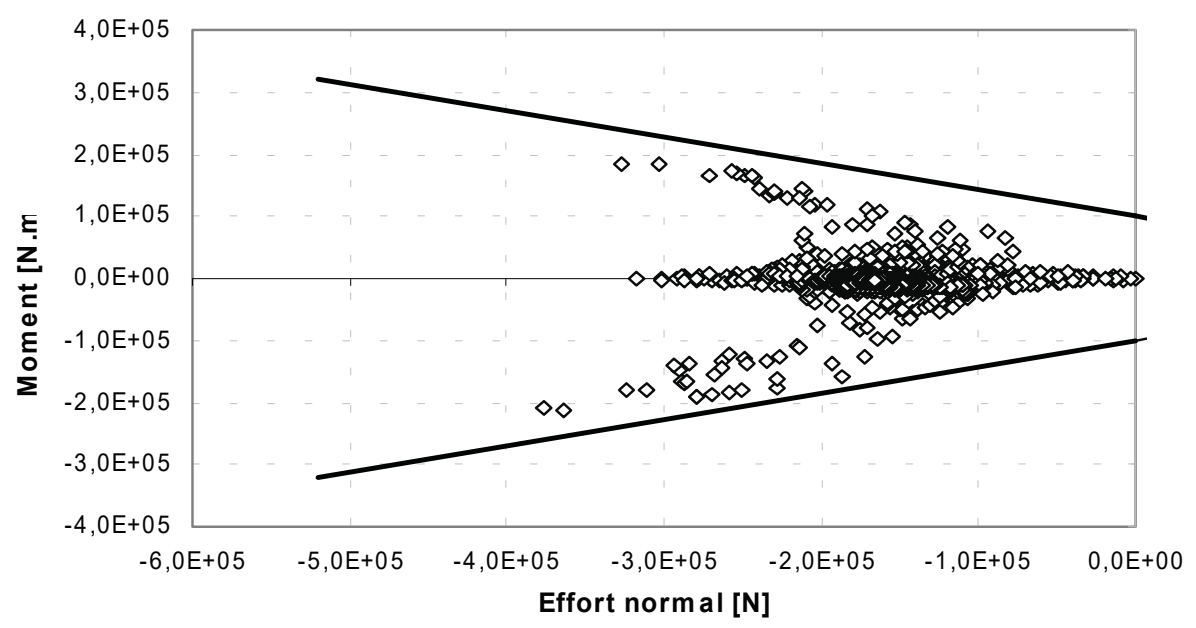

Figure 12. Maquette ECOLEADER. Interaction $M-N$ à la base du niveau 1, mur $X$ gauche (test T3 - résultats numériques)

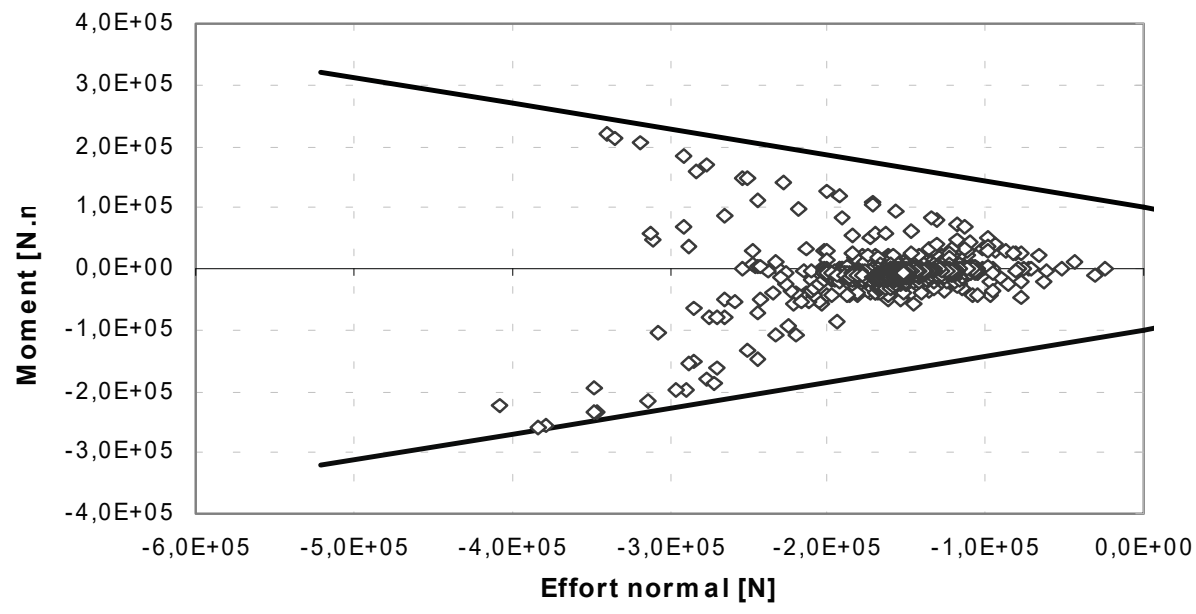

Figure 13. Maquette ECOLEADER. Interaction $M-N$ à la base du niveau 1, mur $X$ gauche (test $T 6$ - résultats numériques)

\section{Conclusion}

Une stratégie de modélisation $3 \mathrm{D}$ simplifiée a été présentée et appliquée à une structure en béton armé soumise à des chargements sismiques (programme ECOLEADER). La structure est modélisée en utilisant des éléments de type poutre multifibre de cinématique Euler-Bernoulli. Nous constatons une bonne reproduction des variables globales dans la direction $\mathrm{X}$ (valeurs maximales et cycles de réponse) 
et la capacité du modèle à prédire la position des zones critiques ainsi que la rupture due à la traction ou à la compression (plastification des armatures, écaillage du béton).

Du fait de sa relative souplesse d'utilisation, ce type de modèle est intéressant pour des études paramétriques afin d'analyser des situations de chargements complémentaires : autres séismes, sollicitation tri-directionnelles... Ce type d'analyse est en cours. Une telle modélisation est un outil utilisable pour l'ingénierie d'autant qu'il est en passe d'être complété par un modèle représentant les effets de l'interaction sol-structure (Crémer, 2001, 2002 ; Grange, 2005).

\section{Remerciements}

Ce programme de recherche a été rendu possible grâce au soutien financier du ministère de 1'Equipement (DRAST-contrat $\mathrm{n}^{\circ} 04$ M6C507), de la Fédération française du bâtiment et de la Commission européenne (programme ECOLEADER d'accès aux grandes installations).

\section{Bibliographie}

Bisch P., Coin A., "The CAMUS 2000 research", $12^{\text {th }}$ European conference on Earthquake Engineering, Paper reference 123, London, 2000.

Bisch P., Coin A., "Seismic behaviour of slightly reinforced walls", $250^{\text {th }}$ anniversary of the 1755 Lisbon earthquake-Proceedings, Lisbon, Portugal, p. 518-522, cd paper no 93, 1-4 November, 2005.

Brachet T., Coin A., Gantenbein F. et al., "Opération CASSBA Conception et analyse sismique des structures en béton armé », Annales de l'Institut technique de bâtiment et des travaux publics, $\mathrm{n}^{\circ}$ 523, Série : Technique générale de la construction, 150, 1994.

CAFEEL-ECOEST/ICONS, Thematic report N.5, Shear Walls Structures, Editors J.M. Reynouard, M.N. Fardis, gen. eds R. T. Severn, R. Bairrão (LNEC), September, 2001.

Coin A., Recherche CAMUS, Rapport final, Présentation générale, Conclusions et perspective, Ministère français de l'Equipement (Plan Génie Civil), Convention 9670011, 2000 .

Cremer C., Pecker A., Davenne L., "Cyclic macro-element for soil-structure interaction: material and geometrical non-linearities", International Journal for Numerical and Analytical Methods in Geomechanics, vol. 25, Issue 13, November 2001.

Cremer C., Pecker A., Davenne L., "Modelling of nonlinear dynamic behaviour of a shallow strip foundation with macro-element", Journal of Earthquake Engineering, vol. 6, p. 175$211,2002$.

Dubé J.F., « Modélisation multicouche des voiles en béton armé », Revue Française de Génie Civil, vol. 1, n 2, juin 1997, p. 285-307. 
Ghavamian S., Mazars J., «Stratégie de calculs simplifiés pour l'analyse du comportement des structures en BA : le code EFICOS », Revue Française de Génie Civil, vol. 2, n 1 , janvier 1998, p. 61-90.

Ghavamian S., Davenne L., Gatuingt F., Elément de poutre multifibre (droite), Fascicule R3.08, Document Code Aster, 2002.

Guedes L., Pégon P., Pinto A., “A fibre Timoshenko beam element in CASTEM 2000”, special publication Nr. I.94.31, JRC, I-21020 Ispra, Italy, 1994.

Grange S., Mazars J., Kotronis P., "Element Soil-Structure interaction under seismic loading: a simplified approach", ALERT Workshop Aussois, 10-12 October, 2005.

Kotronis P., Davenne L., Mazars J., « Poutre 3D multifibre Timoshenko pour la modélisation des structures en béton armé soumises à des chargements sévères », Revue Française de Génie Civil, vol. 8, n² 2-3, 2004, p. 329-343.

Kotronis P., Mazars J., "Simplified modelling strategies to simulate the dynamic behaviour of R/C walls", Journal of Earthquake Engineering, vol. 9, issue 2, 2005a, p. 285-306.

Kotronis P., Mazars J., Nguyen X.H., Ile N., Reynouard J.M., "The seismic behaviour of reinforced concrete structural walls: Experiments and modeling", $250^{\text {th }}$ anniversary of the 1755 Lisbon earthquake-Proceedings, Lisbon, Portugal, p. 441-445, cd paper no 86, 1-4 November, 2005b.

La Borderie C.L., Phénomènes unilatéraux dans un matériau endommageable : modélisation et application à l'analyse des structures en béton, Thèse de doctorat, Université Paris 6, 1991.

Mazars J., "A description of micro- and macroscale damage on concrete structures", Journal of Engineering Fracture Mechanics, vol. 25, n 5-6, 1986, p. 729-737.

Mazars J., Nguyen X.H., Kotronis P., Ile N., Reynouard J.M., Rapport final : Etude sur le fonctionnement sismique de structures à mur à cellules contreventées, Contrat DRAST/Mission Génie Civil, Nº 04MGC 5 07, Rapport final, novembre 2005.

Mazars J., Kotronis P., Ragueneau F., Casaux G., "Using multifiber beams to account for shear and torsion. Applications to concrete structural elements", Computer Methods in Applied Mechanics and Engineering, 2006.

Nguyen X.H., Mazars J., Kotronis P., « Modélisation des structures en béton armé avec des éléments poutres multifibre », $17^{e}$ congrès français de mécanique, Troyes, 29 août-3 septembre, cd numéro 315, 2005.

Owen D.R.J., Hilton E., Finite element in plasticity: Theory and practice, Pineridge Press Ltd, Swansea, England, 1980.

Petrangeli M., Pinto P.E., Ciampi V., "Fiber element for cyclic bending and shear of RC structures. I: Theory", Journal of Engineering Mechanics, vol. 125, $\mathrm{n}^{\circ}$ 9, September, 1999. 